\title{
Lectura e internet: dos tecnologías
}

\author{
Adolfo Rodríguez Gallardo
}

Artículo recibido:

3 de mayo de 2004.

Artículo aceptado:

4 de agosto de 2004.

\section{RESUMEN}

Este trabajo aborda el estudio de la lectura y la escritura como dos tecnologías asociadas que dan origen a un binomio fundamental para el desarrollo de la humanidad, y una tercera tecnología mucho más reciente: Internet. Para ello, se recurre a analizar las innovaciones tecnológicas aplicadas a la bibliotecología y las opiniones que han dado origen a la formación de grupos con posturas divergentes. Se identifican fundamentalmente dos grupos: los tecnólogos y los conservadores. Los primeros cifran sus esperanzas en que la tecnología será la solución a todos los problemas que enfrenta la bibliotecología, en tanto que los segundos se resisten al cambio, sobrevalorando lo existente. Como resultado del embate tecnológico ha surgido la preocupación respecto de la desaparición tanto de la lectura y de la escritura, como de los materiales impresos, de la biblioteca y de la profesión bibliotecaria. Sin embargo aquellos especialistas que han pronosticado 
el desplazamiento de instituciones y profesionales, no han analizado con profundidad las condiciones que deben reunir las tecnologías de la información para satisfacer efectivamente las necesidades de información. La condición fundamental para el uso de las tecnologías de información y comunicación siguen siendo las ancestrales técnicas de lectura y escritura.

Palabras clave: Lectura: Escritura; Internet; Tecnologías de Información y comunicación(TIC).

\section{ABSTRACT}

Reading in Internet: two technologies Adolfo Rodríguez-Gallardo

The paper focuses on reading and writing as two associated technologies that give origin to a fundamental binomial for the development of humanity, and on a much more recent technology: Internet. Thus, an analysis of the technological innovations applied to library science is made, and the opinions that have given birth to the formation of groups with divergent positions are identified. The article centers on two groups: the technologists and the conservatives. The former base their hope on the idea that technology will be the overall solution to library science problems, whereas the latter resist change and over estimate existing means. On the other hand, the technological push has given rise to worries which hold that reading and writing, printed materials, the library and even library science will disappear. However, those specialists who have assumed the displacement of institutions and professionals seem not to have analyzed in depth the conditions that information technologies must fulfill in order to satisfy effectively our information needs. We must not forget that for our technology to operate successfully, man requires dominion of the ancestral techniques of reading and writing.

Keywords: Reading; Writing; Internet; Information and Communication Technologies. 


\section{Acerca de la tecnología}

Ta tecnología es la serie de instrumentos, procedimientos o inventos que U el ser humano ha ideado para hacer fáciles sus actividades diarias: la construcción de grandes obras como presas, termoeléctricas, aeropuertos, rascacielos; el desarrollo de sistemas de comunicación como carreteras, centrales telefónicas, telégrafos, computadoras, satélites; y todos aquellos instrumentos que se emplean para mantener o recuperar la salud, entre muchas otras cosas. La tecnología es la aplicación de los principios científicos, postulados y leyes generales para solucionar determinados problemas; no es la ciencia en sí, sino su enfoque práctico.

Se puede decir que los procedimientos y los instrumentos empleados en el desarrollo tecnológico por sí mismos no amplían ni producen nuevo conocimiento; sin embargo su utilización ha contribuido al desarrollo científico mundial y mejorado con ello la calidad de vida del hombre.

Sería un trabajo titánico ofrecer una lista exhaustiva de las tecnologías que el hombre ha concebido y utilizado a lo largo de su historia, la rueda, la producción del fuego, la imprenta y la computadora, entre millones de otras, son tan sólo algunas de las que han impactado al hombre en su forma de vivir, de relacionarse con otros hombres y, en consecuencia, han afectado también su desarrollo social.

Las tecnologías son de diferentes tipos, algunas utilizan uno o varios instrumentos para su aplicación, como es el caso de la computación; otras hacen uso de una práctica de carácter eminentemente tecnológico, como sucede en el caso de la escritura y la lectura. La transformación de ideas a una forma escrita es una tecnología, en tanto que su lectura, interpretación o decodificación es otra tecnología por sí misma, independientemente de los materiales que se utilicen: tablillas de arcilla, instrumentos cuneiformes, pergamino, tintas, papel, imprenta de tipos móviles, entre otros, hasta llegar a los instrumentos digitales. Estas dos tecnologías le han brindado al hombre la capacidad de utilizar signos, figuras o letras y de transmitir desde conceptos básicos y objetivos - como la descripción física de un objeto, una casa, un árbol, un animal- hasta conceptos abstractos y complejos que incluyen valores como el bien, el mal, la bondad y la justicia, entre otros. Con frecuencia cuando se habla de lectura y escritura no se advierte lo que estos avances tecnológicos han representado para el desarrollo humano, sin ellos la comunicación y transmisión de conocimiento sería mucho más compleja. De hecho algunos historiadores sostienen que es la escritura, y por tanto la lectura, la que le da el carácter de ciencia a su disciplina; hasta antes de la escritura discurría la prehistoria, con su escritura pictórica. La aparición del sistema alfabético de escritura transformó profundamente la organización social y la estructura 
mental del hombre al convertirse en instrumento de la razón y ocupar un lugar preponderante sujeto a reglas discursivas e históricas asociadas al acto de apropiación o lectura. Aunque también hay que señalar que esta idea no es compartida por todos los historiadores.

Entre las tecnologías que se pretenden analizar en este trabajo, están principalmente dos: la primera es muy antigua y ha sido denominada escritura, acompañada de su actividad asociada, la lectura, debido a que forman un binomio fundamental para el desarrollo de la humanidad, ya que sin ellas posiblemente la sociedad no habría alcanzado el grado de desenvolvimiento actual. La segunda es más reciente y resultado de otras tecnologías previamente empleadas como la electrónica y la computación, nos referimos específicamente a Internet; la red de comunicación electrónica de servicios y productos que ha logrado que personas de distintas edades, culturas e intereses, se encuentren en ella y se sirvan de ella para distintos propósitos que van desde la educación, los negocios y la información, hasta la diversión y el esparcimiento.

Con esto no se pretende señalar que las únicas tecnologías que han sido motivo de preocupación de la sociedad y de los bibliotecarios sean las que hoy nos ocupan. Para prestar los servicios que la biblioteca ofrece, ésta ha incorporado muchísimos productos tecnológicos a lo largo de la historia, como el catálogo bibliográfico en forma de libro, y su posterior versión en tarjetas intercalables para mantenerlo al día; y los servicios de foto-duplicación basados en la fotografía o en procesos de tipo xerográfico, por mencionar sólo algunas de las tecnologías empleadas en el trabajo bibliotecario.

En algún momento se ha acusado a la bibliotecología de no ser una ciencia, sino el resultado de la adopción de una serie de procedimientos y conocimientos prácticos que pueden ser identificados como la aplicación de una serie de tecnologías. Por ello es tan fácil pensar que en el trabajo bibliotecario el uso de una nueva tecnología cambia completamente la esencia de la disciplina, cuando lo que realmente modifica son los procedimientos empleados en la prestación de los servicios bibliotecarios. Pero los principios disciplinarios no se ven alterados por las aplicaciones tecnológicas.

Existen profesiones estrechamente relacionadas con el uso de la tecnología, como es el caso de la ingeniería, que tienen como principal actividad la aplicación de ciertos principios matemáticos y físicos a las actividades y necesidades de la sociedad y de los profesionales en ese campo. La bibliotecología, no obstante que está más asociada con las disciplinas humanísticas, ha usado intensamente los productos tecnológicos, desde el inicio de sus actividades y a lo largo de su historia, principalmente en la prestación de los servicios bibliotecarios.

Como algunos autores lo han señalado acertadamente, la escritura es la tecnología inventada por el hombre para conservar ideas y transmitir conceptos 
que permitan la comunicación entre hombres de un mismo lugar o lugares incluso distantes, y de diferentes momentos históricos. La escritura permite transmitir conceptos y mensajes de una gran complejidad, como ya se ha mencionado anteriormente. Escritura y lectura son las bases de todo el quehacer bibliotecario, sin ellas no se podría explicar nuestra actividad ni la existencia de la biblioteca como institución encargada de preservar y transmitir la herencia cultural escrita. Por ello a partir del uso de estas tecnologías los bibliotecarios han ido incorporando los nuevos aportes tecnológicos, los cuales en su momento han sido de gran utilidad, incluso aquellos que se pensó que permanecerían por un periodo más largo del que permanecieron, como sucedió en el caso de la microfilmación.

Por lo anterior el uso de nuevas tecnologías debería ser algo familiar al quehacer bibliotecario y no producir las conmociones que son características en nuestra profesión cada vez que una nueva tecnología empieza a ser utilizada. Deberíamos valorar los nuevos aportes en lo que valen, e integrarlos a nuestra actividad en la medida en que nos son útiles y por el periodo de vigencia que sus características les permitan. Así lo hemos hecho con la incorporación de la máquina de escribir, la fotocopia y los catálogos de tarjetas intercambiables, ¿por qué no hacerlo ahora con Internet?

Ahora bien, la incorporación de toda nueva tecnología enfrenta dos grupos que tratan de hacer prevalecer su enfoque y que luchan entre sí para lograrlo. Por una parte están aquellos que piensan que la nueva tecnología es la solución a todos los problemas y que su aplicación producirá beneficios casi mágicos que en poco tiempo superarán los problemas existentes. Este grupo de personas, integrado por profesionales y no profesionales de la bibliotecología, piensa que la tecnología es lineal y que la nueva substituirá a la que se tiene en un momento dado, y que además ésta desaparecerá y producirá una mejora en los servicios. Como se verá más adelante las tecnologías son complementarias y coexistirán antiguas y nuevas, al menos durante un lapso, y en otros casos quizá para siempre.

El optimismo de este primer grupo los lleva a no apreciar objetivamente los elementos existentes, a menospreciar lo que se ha conseguido y a sobrevalorar la potencialidad de la nueva tecnología, pues se piensa que es el cambio instrumental lo que modifica la esencia del trabajo, cuando en realidad lo que hace es posibilitar su realización de forma más rápida, más precisa y más sencilla. La nueva tecnología ni siquiera garantiza un incremento en la calidad, a menos que ésta rija los servicios y productos previos y que al incorporarse a los procesos se garantice que las actividades que se realizaban antes se ejecutarán ahora con igual o mejor calidad.

El otro grupo se resiste a cualquier cambio y piensa que lo que se ha conseguido hasta ahora, si bien no es perfecto, es, al menos, lo mejor que se tiene 
y se puede lograr. Esta postura tiende a sobrevalorar lo existente y a oponerse a lo nuevo sin analizar las ventajas que pudiera representar la incorporación de nuevas tecnologías o formas de resolver una problemática dada.

Es importante señalar que entre los profesionales de la bibliotecología sucede algo similar. Hay quienes bendicen la llegada de nuevas técnicas y piensan que éstas le proporcionan a la profesión una nueva orientación y sentido, así como que las antiguas formas de trabajar y prestar servicio desaparecerán dejando su lugar a los nuevos servicios derivados del uso de las modernas tecnologías.

Lo que parece perderse de vista es la búsqueda de un equilibrio entre estas dos posturas. El uso de las nuevas tecnologías no garantiza por sí mismo el mejoramiento de los servicios y prácticas bibliotecarias, pero la resistencia a los cambios tampoco nos asegura la superación de los problemas que enfrenta la bibliotecología. Para incorporar las tecnologías apropiadas a cada circunstancia es necesario ejercer un enfoque equilibrado que cuente con un estudio previo a su aplicación, y un programa de entrenamiento de los recursos humanos implicados, avocado a desarrollar las habilidades requeridas para la aplicación efectiva de esas nuevas tecnologías

Más adelante analizaremos en detalle algunas de estas tecnologías, baste decir por ahora que con la incorporación de Internet algunos bibliotecarios pensaron incluso que el nombre de los profesionales debería cambiar por el de ciberbibliotecario.

Por otra parte están quienes en la incorporación de las nuevas tecnologías ven peligros por todos lados: escritura, lectura, libro, publicaciones periódicas, biblioteca y, especialmente, para la profesión misma. Esto resulta de una falta de identidad profesional y de la inseguridad de que los principios y valores de la disciplina son suficientemente fuertes y sólidos como para garantizar la permanencia de la disciplina, la profesión y los bibliotecarios.

Las tendencias imperantes en educación y economía demandan, entre otras cosas, lo que ha dado en llamarse nuevas competencias. Algunas de éstas son realmente primordiales en los escenarios actuales y, otras, aunque no son propiamente novicias, tienen como característica principal que no remplazan a las anteriores.

Pero estas nuevas competencias no substituyen a las habilidades tradicionales; las complementan y las amplían más allá del mundo laboral ${ }^{1}$

Al contrario de lo que se ha sostenido - que la utilización de los instrumentos electrónicos desplazará a las bibliotecas, los bibliotecarios y la lectura-cada 2001) : 16 
día resulta más claro que las tecnologías basadas en el cómputo son especialmente valiosas, pero que no cumplirán las expectativas de aquellos especialistas en tecnologías de la información que han pronosticado tal desplazamiento de instituciones y profesionales. Esta simplificación de los problemas ha sido esgrimida por los especialistas en tecnologías de la información, cuyo enfoque ha consistido en esperar que con sólo incorporar las "nuevas" tecnologías las condiciones existentes también cambien, pero poco o nada se han analizado las condiciones que deben reunirse para que las tecnologías de la información satisfagan efectivamente las necesidades y logren un impacto relevante. Entre estas condiciones se encuentra un entrenamiento que faculte la obtención del mayor beneficio por parte de los profesionales y usuarios involucrados.

Como bien lo han visto Pont y Werquin ${ }^{2}$ en su estudio sobre las características de las nuevas competencias que demandan las actividades económicas, la utilización de la tecnología electrónica y las antiguas habilidades hacen posible la realización de las actividades económicas en un ambiente diferente.

El uso de nuevos instrumentos como Internet requiere que quien los utilice tenga las habilidades y destrezas suficientes para su manejo. Es impensable que alguna persona pueda obtener un beneficio de Internet, del correo electrónico y del flujo global de información empleándolos en actividades económicas, políticas y sociales si no es capaz de leer y escribir fluidamente no sólo en su idioma sino en varios, pues la red contiene información en prácticamente todas las lenguas.

Al contrario de otros profesionales existe un extendido grupo que sostiene que lo que se ha conseguido hasta ahora es mucho y que no se debe cambiar, por lo que se han resistido a la introducción de las computadoras, los catálogos electrónicos, la microfilmación, las fotocopiadoras y, últimamente, a la inclusión de productos y servicios digitales, entre ellos la utilización de Internet.

\section{LA LECTURA}

La escritura misma, que ahora goza de un gran prestigio y de la que nadie pensaría que puede ser dañina, fue considerada, nada más ni nada menos que por Sócrates $^{3}$ como algo perjudicial para el conocimiento, pues no fomentaba el uso de la memoria, que es de donde parte la ciencia. La posibilidad de

2 Ibid, p. 15.

3 Platón, "Fedro o Del amor", en Diálogos, estudio preliminar de Francisco Larroyo (México: Porrúa, 1989), 658-59. 
aprender sin el maestro lleva a la confusión y al error de pensar que se es sabio. El uso de la escritura, y por tanto de la lectura, es un medio para despertar reminiscencias en aquel que conoce ya el objeto del cual se trata, conocimiento adquirido mediante el contacto directo con el objeto de estudio y a través del diálogo en el que el maestro juega el papel central, pues éste elabora las respuestas a partir de las preguntas que se le formulan, y éstas pueden variar debido al intercambio de ideas entre maestro y discípulo. De modo distinto en el discurso escrito siempre se obtendrá la misma respuesta, pues ésta ha quedado inamovible. Un escrito necesita de su autor para defenderse si se ve atacado, pues por sí mismo es incapaz de defenderse, dado que sus argumentos serán los mismos aunque los críticos sean diferentes. La lectura carece de la fuerza creadora que nos ofrece la discusión y el intercambio de puntos de vista, el cuestionamiento individual, pero, sobre todo, el colectivo.

Platón no sólo pensaba que la lectura o el libro, como lo dice en su escrito, eran dañinos para el individuo sino, como lo ha señalado Wick, que también tendría un impacto perjudicial para la sociedad.

Su potencial dañino fue reconocido muy pronto por sus críticos entre quienes se contaba Platón, quien temió al alfabetismo como una fuerza capaz de destruir la unidad interna de la sociedad, desalentando las formas de transmisión oral y por lo tanto desintegrando la experiencia colectiva de sus misterios e ideas culturales. ${ }^{4}$

Así, la lectura fue vista en su momento como un peligro por aquellos que basaban sus formas y métodos educativos en el diálogo y la discusión de las ideas como la mejor forma de educar a las nuevas generaciones. Afortunadamente Platón y Sócrates estaban equivocados y la lectura no se convirtió en un elemento perjudicial para la educación, sino en un instrumento de gran importancia para la transmisión del conocimiento, especialmente entre personas que han vivido en diferentes épocas o que se encuentran en lugares geográficamente distantes, o entre quien escribe y quien lee, pues la lectura posibilita el diálogo entre autores y lectores, contemporáneos o no. Ha sido gracias a la escritura y a la lectura como hemos podido conocer las opiniones de tan ilustres filósofos respecto a esta técnica. Por último la lectura permite poner en contacto a individuos cuyos idiomas son diferentes mediante las traducciones.

En la Edad Media y hasta el Renacimiento la lengua más común para la comunicación entre escritores y lectores era el latín, lo que evitaba el problema que representaba la diversidad de lenguas. Los primeros hombres reconocidos como alfabetizados escribían y leían en latín, no en las lenguas vernáculas 
(las cuales la mayoría de las veces no contaban con un alfabeto propio). El latín era la lengua universal y culta, la lingua franca entre personas instruidas; con la proliferación y fortalecimiento de las lenguas vernáculas, se requirió el surgimiento de alfabetos particulares que representaran las ideas y los sonidos de cada comunidad, lo que posteriormente dio lugar a la necesidad de realizar traducciones entre las lenguas de los emisores y los receptores.

Entre otras muchas cosas la lectura es la tecnología que permite decodificar la escritura, que a su vez es la tecnología que codifica conceptos e ideas. Aunque totalmente imbricadas una en otra, no debemos caer en el falso concepto de que son la misma cosa, pero sobre todo hay que entender que en diversas épocas la sociedad ha permitido y fomentado la lectura; y sin embargo, ha mantenido grandes reservas hacia la escritura por considerarla de mayor peligro para la vida social. Pero posiblemente la definición más simple de lo que son la escritura y la lectura, es aquella que sostiene que son dos tecnologías, como lo ha dicho Graff:

El alfabetismo es ... ante todo, una tecnología o grupo de tecnologías para la comunicación, la decodificación y la reproducción de materiales escritos. ${ }^{5}$

Algunos autores, especialmente quienes se acercan a los puntos de vista de los especialistas en tecnologías de información, han venido sosteniendo que la cultura impresa está en vías de desaparición, e incluso han llegado a sostener que la lectura, o al menos la lectura lineal, está en camino de extinción. Como se puede inferir por la afirmación anterior, las observaciones de los tecnólogos de la información son producto de una sobrevaloración de sus instrumentos tecnológicos y, en el mejor de los casos, una muestra de que no tienen una idea clara de lo que la lectura significa para la humanidad. Es posible que la tecnología substituya algunos recursos tecnológicos de los que se sirve la humanidad, pero es imposible que la lectura sea sustituida, ésta no puede ser suplantada por máquinas, pues aunque tienen muchas ventajas no poseen la capacidad de interpretar y comprender los contenidos de un escrito en el complejo mundo intelectual. La lectura no es sólo una tecnología para tener acceso a la información, es un proceso mental y cognoscitivo que las máquinas no pueden reproducir por más complejas que sean.

Tyner $^{6}$ ha visto con claridad la ambivalencia que implican las tecnologías de comunicación, entre ellas la lectura, en el sentido de que las formas impresas de escritura y lectura se verán dañadas por los medios electrónicos.

5 J. H. Graff, The Labyrinths of Literacy: Reflections on Literacy Past and Present (Pittsburgh, P.A.: University of Pittsburgh Press, 1995), 10.

6 Kathleen Tyner, Literacy in a Digital World: Teaching and Learning in the Age of Information (Mahwah, N. J.: Erlbaum, 1998), 13. 
Así como la cultura impresa no eliminó a la cultura oral sino que le dio nuevas posibilidades de preservar y difundir el conocimiento, de igual forma los medios electrónicos han redimensionado la lectura, ya que, como se analiza más adelante, para hacer uso de los instrumentos electrónicos se requiere de la habilidad lectora. Estas tecnologías operan exitosamente dependiendo del medio ambiente en que se usen y el éxito de su utilización depende del entorno social, cultural, económico y político, porque la lectura es un elemento necesario pero no suficiente para explicar el desarrollo, en tanto que su carencia no explica por sí misma el atraso social. Como se verá más adelante, las ventas de materiales impresos están en los niveles más altos de la historia y lo que los medios electrónicos han producido es la necesidad de reconceptualizar las características o la definición de lo que significa ser alfabetizado.

\section{RADIO Y TELEVISIÓN}

Las expectativas de que la lectura disminuya no son nuevas; el surgimiento de la radio, la televisión y las computadoras hizo creer a muchos que estos instrumentos la remplazarían. La verdad es que ninguna tecnología substituye completamente a otra, ambas conviven durante un periodo dependiendo del beneficio que el instrumento que va a ser sustituido le preste a la sociedad, así como de los aportes y la utilidad que ofrezca la nueva tecnología. Mientras la primera sea de utilidad y resuelva problemas determinados que la segunda no pueda satisfacer, el anterior instrumento permanecerá útil para la sociedad. La aplicación de las tecnologías no siempre es bien recibida por todos, pues hay quienes no las consideran útiles para una determinada situación; el medio en que éstas se implantarán es determinante para seleccionar la tecnología adecuada y sólo en esa situación se verá su aplicación como algo valioso.

Además existen datos que indican que ni siquiera el uso de la radio y la televisión, que emplean tecnología en la que no es necesario leer, - $-\mathrm{y}$ que amplían la posibilidad de recibir información, placer y entretenimiento- ha producido una disminución de la lectura. Un indicio sobre la preocupación causada por el efecto que la televisión tendría en la lectura se halla en el número especial, octubre de 1957, de la revista The Reading Teacher, dedicado íntegramente al tema. En él se analizaban diferentes puntos de vista, pero se concluía que la televisión no substituyó a la lectura y ni siquiera al profesor. La verdad es que la televisión, como en el caso de la BBC de Londres, se ha convertido en aliada de la lectura a través de sus programas de fomento a la misma, así como por los mensajes que continuamente envía para promoverla. En la actualidad a muy pocas personas les preocupa que los niños pasen 
demasiado tiempo frente al televisor y que no lean, lo que les inquieta es que la más nueva de las tecnologías, Internet, substituya a la lectura.

El uso de la radio y la televisión para promover la lectura es descrito con claridad en el documento intitulado Literacy in the World since the 1965 Teberán Conference: shortcomings, achievements, tendencies, es éste un documento preparado por el secretariado de la UNESCO y está incluido en las memorias del Simposio que se celebró en Persépolis, Irán, entre el 3 y el 8 de septiembre de 1975.

Durante varias décadas los medios de comunicación masiva como la radio y la televisión han sido empleados en la lucha contra el analfabetismo, y lejos de un enfrentamiento entre éstos y los medios impresos, se han realizado campañas para promocionar la lectura en dichos medios electrónicos. El incremento de radios (véase figura 1) y televisiones en el hogar no ha disminuido el número de libros publicados, vendidos o tomados en préstamo en las bibliotecas, como se verá más adelante.

Figura 1. Número de radios en las regiones seleccionadas, 1950 y 1970, en millones.

\begin{tabular}{|l|c|c|c|}
\hline \multicolumn{1}{|c|}{ Región } & $\mathbf{1 9 5 0}$ & $\mathbf{1 9 7 0}$ & $\begin{array}{c}\text { Personas por } \\
\text { radio en 1970 }\end{array}$ \\
\hline América Central y el Caribe de habla hispana & 2.7 & 10.5 & 6.3 \\
\hline América del Sur de habla hispana & 4.6 & 17.7 & 7.6 \\
\hline $\begin{array}{l}\text { América Central, América del Sur y el Caribe de } \\
\text { habla inglesa }\end{array}$ & 0.05 & 0.8 & 5.4 \\
\hline Cercano Oriente y Asia del Sur & 1.4 & 17.2 & 40.3 \\
\hline Extremo Oriente incluyendo China & 10.7 & 48.8 & 22.2 \\
\hline
\end{tabular}

Fuente: Harry L. Levin and Robert W. Gillespie, The Use of Radio-Word Neighbors, Oklahoma City, 1971.

Entre la información proporcionada en el Simposio de Persépolis ${ }^{7}$ encontramos que a mediados de la década de 1960, solamente 10 países en desarrollo utilizaban la radio y la televisión como instrumento para combatir el analfabetismo. El ejemplo de estos países se extendió a otras muchas naciones y así, para 1971, ya eran 23 los países que habían incorporado la radio a la lucha contra el analfabetismo, y adicionalmente 12 países informaron que utilizaban la televisión con el mismo fin.

7 "Literacy in the World since the 1965 Teherán Conference: shortcomings, achievements, tendencies, prepared by the UNESCO Secretariat", en A Turning point for literacy: the spirit and declaration of Persepolis: proceedings of the International Symposium for Literacy, Persepolis, Iran 3 to 8 September 1975, edited by Leon Bataille, (Oxford: Pergamon, 1976), 22. 
Estos datos nos permiten constatar que los distintos medios no son excluyentes sino complementarios, y que el planteamiento de que una tecnología sustituirá a otra es, cuando menos, poco riguroso, pues si en el fondo los medios electrónicos no substituyen a la producción impresa, menos podrán desplazar a la lectura como forma de acceder al conocimiento y la comunicación.

Es frecuente que los padres busquen la mejor educación para sus hijos y que entre los mitos que se cuentan sobre la educación del futuro esté el de que éste nos depara la desaparición de los medios escritos, de la biblioteca y de la lectura. Como ha sostenido Tyner este razonamiento es inexacto, pues parte de un supuesto falso que es creído por los padres, los maestros y los alumnos, y que sostiene que la vida de un estudiante cambia con el uso de la tecnología de información y comunicación. Es frecuente escuchar que las tecnologías de comunicación e información son recursos poderosos que aumentan las capacidades de los individuos. Pero parece más complicado que eso, pues no todos los que las usan alcanzan el éxito, se requieren ciertas características esenciales para lograrlo.

El éxito lo logran aquellos que entienden y manipulan al menos uno de los dos principales campos de las tecnologías de la información: (a) la infraestructura de la información técnica, esto es, las propiedades y materias primas del alfabetismo, incluyendo las herramientas, el personal técnico, el diseño de sistemas y los mecanismos de distribución; y (b) la información en sí misma, o más precisamente, el estilo discursivo que es característico de cada medio y que le da forma a su contenido, sintetizado brevemente por Marshall McLuhan en la simple frase de "el medio es el mensaje”. Lo anterior tiene que ver con la producción de información que es análoga a la escritura. ${ }^{8}$

El uso de Internet ha generado la reacción de muchas personas que le atribuyen características dañinas, pero al mismo tiempo otros la han visto como un instrumento que resolverá todos los diferentes problemas en su totalidad.

Se le atribuye a Internet, entre otras cualidades, virtudes y posibilidades, que su uso permitirá solucionar los rezagos educativos y culturales, elevar la educación, satisfacer la curiosidad en todos los sentidos, establecer comunicaciones más rápidas y eficientes, y aumentar el comercio y la actividad económica en general, entre otras cosas. Pero también se ha dicho que su uso generalizado, además de alejar a los jóvenes de la lectura, los conduce a la utilización de materiales que les producen daño mental y físico, y que en ocasiones los hace objeto de perversión y hasta de prácticas diabólicas. Todo esto es dicho sin ningún argumento de peso, claro que Internet puede ser eso 
y muchas cosas más. Pues incluso se ha dicho que el uso de Internet provoca que los estudiantes lean menos, consulten menos fuentes bibliográficas, pasen demasiado tiempo en juegos poco educativos y, además, que Internet provoca baja concentración y que los niños, al pasar tanto tiempo enfrente de la computadora, incluso dejan de hacer el ejercicio que su organismo requiere y los hace propensos a la obesidad. Todo esto y muchas cosas más se podrían decir también de la comunicación escrita, libros, folletos y publicaciones periódicas. El uso inadecuado de estas cosas causa que los niños no hagan ejercicio, que no se concentren en sus tareas escolares por estar leyendo libros que encuentran fascinantes y que estimulan su deseo de concluirlos. Finalmente no son los medios los que provocan los efectos perniciosos, sino la utilización que hagamos de ellos.

Sin embargo Tyner ${ }^{9}$ señala que estas observaciones son infundadas, ya que ninguno de quienes sostienen estos puntos de vista aceptarían como cierta la afirmación de que los niños lectores son más gordos que los no lectores, o que los niños lectores tienen peor condición física que los no lectores.

\section{INTERNET}

Dentro de las actividades de cómputo se encuentra Internet, que es una red de redes de computadoras que enlaza a millones de computadoras de diferentes tamaños, marcas y lugares, pero más importante aún, que hace posible su utilización como herramienta de comunicación, intercambio de información, explotación de bancos de información y trabajo en equipo. Los propósitos para los que se usa este instrumento son variados y posiblemente los más conocidos sean el correo electrónico, la búsqueda de información, la diversión, las actividades económicas de diversos tipos como son las relacionadas con operaciones bancarias como depósitos, pagos y transferencias; pero también la adquisición de acciones en las bolsas de valores, un creciente comercio electrónico que incluye la adquisición, entre otros productos, de boletos de avión, así como las labores administrativas de instituciones públicas y de gobierno; la tramitación o renovación de solicitudes de servicios, y en escuelas la inscripción, altas y bajas de cursos y el conocimiento de los historiales académicos. Y muchas otras más que sería interminable y tedioso enumerar y que no es necesario hacer porque no es la finalidad de este trabajo enlistar todas las posibilidades que ofrece el uso de Internet.

En el campo de la información usar Internet es especialmente importante, pues permite que estudiantes y profesores interactúen, y que colegas con 
intereses comunes se comuniquen para intercambiar experiencias y enriquecer mutuamente sus trabajos. Lo cual se puede hacer con gran efectividad mediante el uso del correo electrónico.

Otra posibilidad de uso es localizar información tanto en bancos de datos bibliográficos y documentales que contienen producción técnica y académica de alto nivel, como buscar en la red algunas posibilidades adicionales, pero siempre y cuando se sepa cómo buscar.

Para la finalidad de este trabajo es necesario que analicemos lo que se ha dicho sobre lo que puede hacer Internet, lo que se dice que va a producir, y lo que realmente ha pasado, al menos en estos últimos años. Evidentemente en esta era de cambios tan acelerados no tenemos la posibilidad de predecir cómo evolucionará Internet, sólo podemos prever para unos pocos años.

Se ha dicho que Internet cambiará las formas de leer y que los lectores ya no pasaran tanto tiempo haciéndolo en forma lineal, sino que podrán hacerlo en otra forma, especialmente con el uso de hipertextos. ${ }^{10}$ La lectura lineal es esencial para comprender el significado de una frase, oración o enunciado. La lectura no lineal es adecuada para la búsqueda de información documental, pero para poder interpretar el contexto de los términos buscados, así como el significado de los conceptos en los cuales están inmersos los términos, es necesario e indispensable hacer una lectura lineal.

También se ha dicho que la lectura desaparecerá como consecuencia de esta nueva tecnología y que en el futuro toda comunicación será de carácter visual. Hay que aclarar que un punto de vista como éste no ha sido sostenido ni siquiera con respecto de la televisión, que es un medio mucho más visual que Internet, por lo que parece una exageración pensar que el futuro será predominantemente visual.

En realidad, las actividades que se presentan relacionadas con la lectura y la escritura, como el correo electrónico y la búsqueda de información, no han experimentado una disminución; al contrario, con el empleo del correo electrónico se está escribiendo más que antes y ya no se depende solamente del teléfono para una comunicación rápida. El correo electrónico permite además imprimir el mensaje para su posterior estudio y análisis o bien para discutirlo y compartirlo con otras personas; pero sin escritura y lectura el correo electrónico simplemente no se puede dar. La modalidad de "chatear" (mediante la utilización de la computadora) se puede hacer verbalmente, pero la mayoría de los usuarios de Internet lo hacen de forma escrita y por tanto deben tener un adecuado nivel de comprensión de la lectura.

La búsqueda de información se hace mediante la lectura de los materiales que nos ofrece Internet y no es posible encontrar información pertinente y 
valiosa sin leer el contenido de lo que se encuentra, sin hacer búsquedas de términos relacionados entre sí, o sin entender que existen referencias lingüísticas o temáticas.

Más bien lo que ha sucedido con estos instrumentos electrónicos y digitales es que lejos de disminuir la lectura la han impulsado, y que continuarán haciéndolo porque es necesario o, mejor dicho, indispensable leer para realizar un trabajo exitoso en Internet. No se puede tampoco asegurar que estos hechos no hayan provocado que algunas personas dejen de utilizar la lectura en forma intensiva, pero lo más seguro es que tampoco la usarían si no tuvieran Internet a su disposición.

Hasta el momento, los miedos acerca de que el uso de las tecnologías de la información substituirían a las tecnologías existentes en la prestación de productos y servicios han sido, por decir lo menos, una exageración. A continuación analizaremos alguna información que confirma que, lejos de substituirla, Internet ha venido a complementar la lectura de material impreso.

\section{INTERRELACIÓN ENTRE LECTURA E INTERNET}

Petrucci nos proporciona datos sobre tres aspectos importantes que nos ayudan a evaluar la lectura, la producción de libros, la producción de periódicos y el préstamo de libros. Permítasenos citar en extenso a este autor, pues los datos que nos ofrece son importantes:

c) La producción de libros crece vertiginosamente en todo el mundo, tanto en los dos países gigantes, EE UU y la URSS (al menos hasta 1989), como en Europa, como en los países pertenecientes a otras áreas (pero sólo a partir de la última década). En 1975 fueron producidos en el mundo 572.000 títulos; en 1980, 715.000; en 1983, 772.000. A principios de los ochenta, Europa, con un 15 por ciento de la población, producía aún el 45,6 por ciento de los libros: la URSS, con 8,1 por ciento de la población, el 14,2 por ciento y Estados Unidos, con el 7,5 por ciento de la población, el 15,4 por ciento. Este cuadro está destinado a cambiar en el futuro, pero no de un modo radical, ni excesivamente rápido.

d) Por lo que respecta a la prensa, en 1982 se producían en todo el mundo 8,220 periódicos, de los cuales 4.560 en los países desarrollados (en USA, 1815) era muy abundante la circulación de ejemplares en países con una antigua tradición de lectura y de información: en Gran Bretaña se contaban 690 ejemplares por cada mil habitantes; en Japón, 751; en Suecia y Alemania del Este, 496; y en Francia, 205.

e) Los préstamos de libros efectuados en las bibliotecas públicas proporcionan datos análogos. Según el cómputo de 1980, Estados Unidos está a la cabeza 
con 986 millones de volúmenes, seguido de la URSS, con 665 millones, y por Gran Bretaña, con 637; lo que quiere decir que, dado el porcentaje de la población, este último es el país en el que la circulación librera por la vía del préstamo es la más alta del mundo. Le siguen Francia con 89 millones, Dinamarca con 79 y Suecia con 77; pero para estos dos últimos países valen las mismas consideraciones que hemos planteado para Gran Bretaña. ${ }^{11}$

Son varios los aspectos que merecen destacarse de los datos proporcionados por Petrucci. El primero es que los datos no son absolutos y que tienen que ser ponderados tomando en cuenta la población de los países. Otro aspecto es que si bien el número de periódicos disminuyó, no así el tiraje de éstos, lo que representa no una baja en el número de lectores sino un decremento en la oferta de diarios, con lo cual se ve afectada la diversidad de posibilidades de leer, pero no así el monto de lectores y el tiempo dedicado a la lectura.

En 1991, Graff publicó un trabajo en el que nos presenta un panorama ambivalente, por una parte una gran producción de libros, sobre todo en los países desarrollados, y por la otra una falta de materiales bibliográficos en los países en vías de desarrollo. Como ejemplo señala que cada minuto se publica un nuevo libro, lo cual implica que diariamente se publican 1440 libros nuevos, y añade que los tirajes de cada obra son diferentes dependiendo del tema, el país en el que se edita el libro, el formato y otros aspectos más. No obstante, se publican ocho mil millones de volúmenes cada año, la mayor parte de los cuales se concentra en los países desarrollados. En estos países se dan casos de sobreproducción, lo que implica que se producen más títulos y volúmenes de los que el mercado demanda en tanto que existen países que carecen por completo de material bibliográfico. Los precios de los materiales bibliográficos se convierten en un elemento que impide que los países en desarrollo hagan uso de los libros producidos en los países desarrollados. En los países en desarrollo la carencia de una industria editorial es otro problema al que también hay que hacer frente, y por último tenemos la barrera del lenguaje. Muchos países que tienen lenguas locales se encuentran con la dificultad de producir una adecuada bibliografía debido a su diversidad idiomática.

El mismo autor nos presenta una serie de estadísticas que prueban la paradoja de que en los países desarrollados aquellos que pueden leer no lo hacen.

En Italia y Hungría por ejemplo, 40\% de la población no lee de modo significativo, en Francia 53\% no lo hace, y en los Estados Unidos con los niveles más bajos

11 Armando Petrucci. "Leer por leer: un porvenir para la lectura", en Historia de la lectura en el mundo occidental. Bajo la dirección de Guglielmo Cavallo y Roger Chartier, (Madrid: Taurus, c1998), 596-597. 
de analfabetismo absoluto, el analfabetismo "funcional" es muy alto, llegando la estimación al $50 \%$ de los adultos. ${ }^{12}$

Otra información en el mismo sentido es la reunida y publicada por la Benton Foundation ${ }^{13}$ en 1996, en ese trabajo se afirma que existe una correlación entre el uso de las bibliotecas, la asistencia a las librerías y el uso de computadoras, y que lejos de excluirse se complementan una a la otra. De aquellos que asistieron a la biblioteca al menos una vez al año, el 88 \% también fue a la librería al menos una vez en el mismo periodo. Este porcentaje es muy alto y muestra cómo los lectores buscan sus materiales de lectura en la biblioteca y en la librería.

De quienes tienen una computadora en casa y que, siguiendo el planteamiento simplista encontrarían todo en Internet, el 79\% asistió a la biblioteca y el $90 \%$ fue a la librería al menos una vez al año. Pero lo que es más importante, la posesión de la computadora fortalece la asistencia a la librería y a las bibliotecas; de aquellos que no poseen una computadora solamente el 60\%, esto es 19 puntos porcentuales menos que cuando sí la tienen, asistió a una biblioteca; y el 69\%, o sea 21 puntos porcentuales menos que los propietarios de una computadora, acudieron a la librería. Claramente, las formas electrónicas no han desplazado al libro sino que han fortalecido su uso.

Para concluir con este tema encontramos la opinión de Klaus Ring ${ }^{14}$ sobre las técnicas empleadas en la impresión de materiales bibliográficos, en especial las utilizadas en la producción de libros, las cuales no se han mantenido estáticas, más bien desde hace 500 años han sufrido modificaciones que las han hecho mejores y más productivas. Los cambios en la tecnología de impresión en papel han sido tan impresionantes que han facilitado la calidad de su impresión, como poder atender las necesidades de formatos y grandes tirajes que se demandan en la actualidad. La tecnología de impresión ha sido una de las más cambiantes y ha ido incorporando los medios disponibles en cada momento: fotografía, transmisión de textos e imágenes por cable, offset, utilización de las tecnologías de la información. La impresión nos muestra cómo los avances tecnológicos pueden ser incorporados exitosamente a una tecnología de más de 500 años de antigüedad.

Toda esta información puede ser complementada con los datos publicados por el Washington Post en referencia a los bienes y productos que los adultos

12 Harvey J. Graff, The Literacy Myth: Literacy and Social Structure in the Nineteenth Century, (New Brunswick, N. J.: Transaction Publishers, c1991), 2.

13 Kathleen Tyner, Literacy in a Digital World, p. 40.

14 Klaus Ring, "Are the Internet and printed products interchangeable media?", en World Library and Information Congress: 69th IFLA General Conference and Council, 1-9 August 2003. Berlin. Disponibilidad < http://ifla.org/IV/ifla69/papers/139e_trans-Ring.pdf > [Fecha de consulta: 25 Marzo 2004], 7. 
del área de Washington, D. C. obtienen mediante el uso de Internet. Se encontró que el 49\% de los adultos hacen compras vía electrónica y que el 22\% de los bienes que adquieren son libros, seguidos por boletos de aerolíneas con 21 $\%$, CDs, cintas u otra música con $14 \%$, ropa y accesorios $14 \%$, programas para computadora $9 \%$, flores $8 \%$, computadoras $7 \%$, y juegos y juguetes $7 \% .{ }^{15}$

El mismo periódico informó que las compañías que se valen de Internet y que tienen mayores ingresos presentan características interesantes. La información presentada no incluía a algunas de las principales compañías, pues éstas no habían reportado sus ventas en el momento de publicar la información, como era el caso de USA Interactive, Expedia, Hotels.com y WedMed. Los resultados son reveladores cuando se observa que los principales productos de venta son libros. De las compañías reportadas la que más ingresos obtuvo fue America on Line de AOL Time Warner con 2,197 millones, seguida por Amazon.com con 1,084 y posteriormente MSN (División de Microsof) con 508 millones. ${ }^{16}$

Estas cifras nos indican que la utilización del Internet no ha provocado en forma generalizada un decremento de los lectores, o al menos de los compradores de libros. Al contrario, Internet fomenta la lectura y el uso del correo electrónico, y muchas de las transacciones que se realizan en ella sólo se pueden hacer exitosamente si el usuario lee y comprende las indicaciones y los pasos que debe seguir.

Klaus Ring ${ }^{17}$ llega a conclusiones parecidas en el trabajo presentado ante el 69 Congreso Mundial de Bibliotecas y de Información, en Berlín, al decir que el número de lectores intensivos ha aumentado en Alemania en los últimos años, y que estos lectores tienen una actitud más dinámica ante la lectura. Por una parte estos lectores leen más libros y por la otra compran más libros. Enfatiza que las novelas — que juegan un papel importante en la promoción de la lectura- y los materiales educativos han incrementado el número de lectores intensivos.

Sin embargo los datos que se nos presentan son en ocasiones contradictorios y nos llevan a pensar que mucha de la información disponible es muy reciente y que puede contener imprecisiones. El periódico Reforma, ${ }^{18}$ de la ciudad de México, publicó el 16 de septiembre de 2002 una encuesta posiblemente realizada por el propio periódico, pues no se señala la fuente, en

15 Nancy McKeon. “Online shopping blossoms”, en The Washington Post, 8 December 2002, H5.

16 "Internet giants", en The Washington Post, 1 May 2003, E7.

17 Klaus Ring "Are the Internet and printed products interchangeable media?" 3.

18 "Economía y finanzas/sondeo," Reforma [En línea] Disponibilidad: http://www.reforma.com/general/sondeos/resultado.asp? [Fecha de consulta: 16 Septiembre 2002]; y "Negocios/sondeo," Reforma [En línea] Disponibilidad:

http://www.reforma.com/sondeos/negocios/default.html [Fecha de consulta: 16 Septiembre 2002] 
la que indicaba que el $81 \%$ de los que usan Internet lo hacen para buscar información; otro 11\% lo hace para "chatear"; $85 \%$ buscan entretenimiento y nadie lo usa para hacer compras. En esa misma encuesta se ofrecía la oportunidad de consultar encuestas anteriores y al hacer esto los resultados fueron sorprendentes porque sin indicar la fecha en que se realizó la anterior encuesta, se encontró que la población usuaria de Internet lo hace para dos actividades que corresponden al 50\% cada una, buscar información y buscar entretenimiento. La consistencia entre las dos encuestas es que los usuarios buscan información y ésta sólo se puede lograr mediante la lectura, lo que significa que Internet, lejos de ser una amenaza para la lectura, se convierte en un excelente aliado para su fomento.

Los aspectos tratados anteriormente contradicen las predicciones de que la biblioteca, los bibliotecarios y la lectura están en camino de desaparecer. Nancy Kranich ${ }^{19}$ escribió en American Libraries un mensaje que intituló "Why Libraries are More Popular than Ever". (Por qué las bibliotecas son más populares que nunca) y en el que señala que según datos de la American Library Association 81\% de los estadunidenses usan las bibliotecas todos los años, cifra muy elevada si la comparamos con la utilización de la biblioteca en otras partes del mundo. Este elevado número de lectores tiene un fuerte impacto en las políticas públicas, y si bien es cierto que entre los que favorecen el uso de la biblioteca se considera que lo logrado es suficiente, habría que aclarar que el tema del apoyo a la biblioteca es una constante en la vida política norteamericana. En los seis años previos a la publicación del artículo mencionado se gastaron tres millones de dólares en actualizar las bibliotecas, y el porcentaje de bibliotecarios de consulta se incrementó en 56\%.

La utilización de las bibliotecas públicas ha demostrado tener un impacto importante en el nivel de calidad con que se realiza la lectura. Parece haber una estrecha relación entre la existencia de la biblioteca pública, el elevado uso de ésta y la calidad de lectores. Kranich considera que tanto las bibliotecas como las librerías están disfrutando de un renacimiento y que este beneficio es compartido por Internet. Esta información confirma el punto de vista de que las tecnologías y otros recursos son complementarios y no sustitutivos.

La relación entre lectura y tecnologías de información debe ser de colaboración, los especialistas en cómputo no deben suponer que los recursos electrónicos y sus habilidades solucionarán todos los problemas, ni que con su trabajo substituirán los conocimientos, las tecnologías y las habilidades existentes.

Para poder hacer un uso efectivo de la carretera de la información se debe leer, y leer bien, ${ }^{20}$ esto significa que se debe poseer la destreza para abordar

19 Nancy Kranich. "Why libraries are more popular than ever", en American libraries (April 2001), 7.

20 Klaus Ring, "Are the Internet and printed products interchangeable media?" 4 
todo tipo de material escrito: artículos, crónicas, libros, etcétera. Por eso son importantes los resultados del estudio PISA, en el que se encontró que para hacer un uso eficiente de las nuevas tecnologías de la información se requiere tener un elevado manejo del lenguaje y una alta capacidad lectora. De tal suerte que la tecnología de la comunicación y la información no son alternativa frente a la necesidad de aprender a leer a temprana edad y ser capaces, mediante la lectura, de mejorar los niveles de comprensión y aprendizaje, así como de mejorar la habilidad de obtener información por sí mismos.

Es importante que los planteamientos que se hacen al incorporar nuevas tecnologías tengan el necesario respeto por los profesionales, las disciplinas e instituciones que han venido trabajando por mucho tiempo, en ocasiones por siglos, en los diversos campos del conocimiento. Las actitudes prepotentes de los especialistas en tecnologías de la información no ayudan en nada a realizar un trabajo constructivo, aunque finalmente éste se logra pero sólo después de tener que presentar una lucha innecesaria entre dos corrientes. Tampoco ayuda mucho al trabajo constructivo la inseguridad con la que algunos profesionales de la bibliotecología observan las nuevas propuestas tecnológicas, y el miedo con que trabajan ellos mismos hace que no valoren su trabajo y tradiciones, lo que dificulta la comunicación.

Ninguna tecnología puede substituir los principios y valores de los servicios bibliotecarios, incluida aquí la lectura, por ello es menester que cuando enfrentemos las nuevas tecnologías no veamos en ellas una amenaza, sino que pensemos cuál es la mejor forma de servirnos de ellas para seguir cumpliendo con los objetivos de nuestra disciplina y nuestro trabajo profesional. La clave no está en la confrontación, sino en la integración de lo antiguo y valioso con lo nuevo y vigoroso que el avance tecnológico nos ofrece.

\section{CONSIDERACIONES FINALES}

La nueva tecnología no tiene como finalidad reemplazar a la que ya existe, más bien se propone complementar a las existentes y facilitar la realización de muchas actividades humanas. Los temores de Sócrates y de los bibliotecarios del siglo XX sobre las nuevas tecnologías eran infundados. El diálogo como método de estudio de las cosas y los fenómenos humanos sigue existiendo, la escritura y la lectura no han hecho sino fortalecerlo. La lectura no se ha visto amenazada por el uso de las computadoras, ni por el Internet; al contrario, su uso ha requerido que los usuarios sean cada vez más aptos para la escritura y la lectura, sólo así podrán enfrentar la avalancha de información que los nuevos instrumentos electrónicos ofrecen. Ambas tecnologías, como otras miles más, les han permitido a los seres humanos enfrentar de 
mejor forma sus problemas y, en el caso particular de la lectura y el Internet, los problemas del conocimiento.

\section{Obras CONSUltadas}

Bataille, Leon, ed. A Turning point for literacy: the spirit and declaration of Persepolis: proceedings of the International Symposium for Literacy, Persepolis, Iran 3 to 8 September 1975. Oxford: Pergamon, 1976. viii $+279 \mathrm{p}$.

Birkerts, Sven. The Gutenberg elegies: the fate of reading in an electronic age. Boston, Mass.: Faber and Faber, 1994. x +231 p.

Eisenstein, Elizabeth L. "The Emergence of print culture in the West." En Journal of Communication 30, no. 1 (Winter 1980): 99-106.

Gilmont, Jean-François, ed. The Reformation and the book. English edition and translation by Karin Maag. Aldershot, Hants, England; Brookfield, USA: Ashgate, 1998. xxii + 498 p.

Graff, Harvey J. The Labyrinths of literacy: reflections on literary past and present. Rev and expanded ed. Pittsburgh: University of Pittsburgh Press, c1995. xxxiii +349 p.

- - The Literacy Myth: Literacy and Social Structure in the Nineteenth Century. New Brunswick, N. J.: Transaction Publishers, c1991. xliv $+352 \mathrm{p}$.

Hiltz, Starr Roxanne and Murray Turoff. The network nation: buman communication via computer. With foreword by Suzanne Keller. Rev. ed. Cambridge, Mass.: MIT Press, 1993. xxxi + 557 p.

Iannone, Patrick V. "Just beyond the horizon: writing-centered literacy activities for traditional and electronic contexts", en Reading Teacher 51, no. 5 (February 1998): 438-443.

"Internet giants" The Washington Post, 1 May 2003, E7.

Kranich, Nancy. "Why libraries are more popular than ever", en American libraries (April 2001) : 7.

Lafuente López, Ramiro. Biblioteca digital y orden documental. México: UNAM, CUIB, 1999. 100 p.

Leu, Donald J. "Internet Project : preparing students for new literacies in the Global Village", en Reading Teacher 54, no. 6 (March 2001): 568-572

McKeon, Nancy. "Online shopping blossoms”, en The Washington Post, 8 December 2002, H5.

McLuhan, Marshall. The Gutenberg Galaxy. [Toronto]: University of Toronto Press, [1962]. 293 p.

Manguel, Alberto. A bistory of reading. New York: Penguin Books, 1997. $372 \mathrm{p}$.

Negroponte, Nicholas. Being digital. New York: Alfred A. Knopf, 1995. $243 \mathrm{p}$. 
Ong, Walter J. "Literacy and orality in our times", en Journal of Communication 30, no. 1 (Winter 1980): 197-204.

Platón. The collected dialogues of Plato, including the letters. Edited by Edith Hamilton and Huntington Cairns, with introd. and prefatory notes, [Translators: Lane Cooper and others]. New York: Pantheon Books, [1961]. xxv + 1743 p.

- - "Fedro o del amor", en Diálogos. Estudio preliminar de Francisco Larroyo 623-661. México: Porrúa, 2000.

Pont, Beatriz and Patrick Werquin. "How old are new skills?", en $\mathrm{Ob}$ server no. 225 (March 2001): 15-17

Rheingold, Howard. The virtual community: homesteading on the electronic frontier. Reading, Mass.: Addison-Wesley, c1993. viii + 325 p.

Rin, Klaus. "Are the internet and printed products interchangeable media?", en World Library and Information Congress: 69th IFLA General Conference and Council, 1-9 August 2003. Berlin.

Disponibilidad

<http://ifla.org/IV/ifla69/papers/139e_trans-Ring.pdf> [Fecha de consulta: 25 de marzo de 2004].

Sanders, Barry. A is for ox: violence, electronic media, and the silencing of the written word. 1st ed. New York: Pantheon Books, c1994. xiii $+269 \mathrm{p}$.

Selfe, Cynthia L. Technology and literacy in the twenty-first century: the importance of paying attention. Carbondale, Il.: Southern Illinois University, 1999. xxiii $+182 \mathrm{p}$.

Stoll, Clifford. Silicon snake oil : second thoughts on the information bighway. New York : Doubleday, 1995. 247 p.

Tyner, Kathleen. Literacy in a digital world: teaching and learning in the age of information. Mahwah, N. J.: Erlbaum, 1998. xii + 291 p.

Wick, Tom. "The pursuit of universal literacy", en Journal of Communication 30, no. 1 (Winter 1989): 107-12. 